\title{
Bitcoin's energy consumption and social costs in relation to its capacity as a settlement layer
}

\author{
Lennart Ante ${ }^{1}$, Ingo Fiedler ${ }^{1,2}$ \\ ${ }^{1}$ Blockchain Research Lab, Max-Brauer-Allee 46, 22765 Hamburg \\ ${ }^{2}$ Concordia University, Faculty of Arts \& Science, Montreal, Canada
}

\begin{abstract}
Bitcoin runs on energy. The decentralized network's amount of energy consumption has resulted in multifaceted discussions about its efficiency and environmental impact. To put Bitcoin's energy consumption into perspective, we propose to relate (a) the energy consumption in TWh and (b) resulting social costs in the form of carbon emissions to the Dollar value settled on the Bitcoin network. Both metrics allow to relate and quantify the capacity of Bitcoin as a settlement layer to the network's energy consumption and resulting carbon emissions, or social costs. We find that in early 2021 Bitcoin (a) settles between $\$ 2,333$ and $\$ 7,555$ for each Dollar spent on energy and (b) that, on average, a Dollar settled on the Bitcoin blockchain causes in social costs between $0.007 \%$ and $0.01 \%$, depending on the estimated energy consumption converted into the costs of carbon emissions. These results help to assess the efficiency, cost and sustainability of Bitcoin and may allow a comparison of Bitcoin with existing settlement base layers such as Fedwire or gold.
\end{abstract}

\section{Introduction}

In early 2021, the market capitalization of all Bitcoins [1] exceeded $\$ 1$ trillion [2]. Over the course of the year 2020 , over $\$ 6.3$ trillion were moved on the decentralized Bitcoin network, which corresponds to a value of about $\$ 17.2$ billion per day. In the first two months of 2021 alone, this value amounts to over $\$ 80$ billion per day and extrapolates to an annual value settled of over $\$ 29$ trillion [3]. Putting this figure in the context of, e.g., the centralized foreign exchange market, which is estimated at a daily volume of $\$ 6.6$ trillion [4], the Bitcoin network would represent $0.26 \%$ of this market. If compared to a payment service such as VISA, Bitcoin's annually settled value of $\$ 29$ trillion would represent roughly 2.5-times the annually value settled on the VISA network [5]. However this much-used comparison with VISA that works on top of an existing base-layer settlement system is not particularly apt. In fact, a better comparison is to other base-layer settlement systems like the American clearing house Fedwire, which in 2020 processed about 727 thousand transactions per day with an equivalent value of $\$ 3.3$ trillion [6].

A key characteristic of the Bitcoin blockchain is that the underlying security of the decentralized network is ensured by the proof-of-work consensus mechanism [7]. In this process, so-called miners expend computing capacity by trying to guess a desired string consisting of numbers and letters before other miners, whereupon a reward, the so-called block reward, is paid out to the miner that successfully guessed the string. This "guessing" process does not follow any clear logic, but works via repetitive trial and error [8]. Accordingly, Bitcoin runs on energy, which in turn led to much discussion and criticism [9].

Various studies have set the goal of quantifying the energy consumption and resulting carbon emissions of the
Bitcoin network [10]-[15] and other proof-of-work blockchains [16], [17]. This has even led to public scientific debates on the validity of specific models, assumptions or implications. With the Cambridge Bitcoin Electricity Consumption Index (CBECI) [18] and the Bitcoin Energy Consumption Index (BECI) [19], two scientifically sound methods have emerged that publish daily estimates of the energy consumption of the Bitcoin network. As of March 2021, the CBECl estimates an annual electricity consumption of 128 terawatt hours (TWh). At 78 TWh, the $\mathrm{BECl}$ 's consumption estimate is significantly lower. Regardless of which of these two values is closer to the actual power consumption of the network, it can be clearly stated that it is a quantity of international significance, as it would mean that the Bitcoin network accounts for about $0.34-0.57 \%$ of the global energy consumption of 22,315 TWh in 2018 [20].

While the energy consumption of the Bitcoin blockchain is undoubtedly "high", it needs to be understood why this energy is expended and what benefit is created from the expenditure. The question of why is easily explained. Miners on the network receive transaction costs and newly issued Bitcoins when they successfully solve the proof-of-work process and validate unconfirmed Bitcoin transactions [21], [22]. Accordingly, the why can be explained via a simple monetary incentive. Miners compare the expected monetary reward with their (electricity) costs and are active as long as it is profitable. To put it simple: If the price of Bitcoin increases and the price of energy remains the same, the amount of energy a miner is willing to spend increases accordingly. The much more complex question of benefits from this energy the topic of this article. The essential use of Bitcoin is to make digital values-irrespective of the fact whether it represents "digital currency" or "digital gold"-secure and transfer- 
able without the need of intermediaries. Effectively, intermediaries and their associated costs are replaced by an energy fueled protocol [8].

A first rationale for the significant energy consumption is the secure storage of value. In this paper we focus on the second rationale of Bitcoin: the settlement layer. With a daily settlement equivalent of roughly $\$ 80$ billion as of 2021, the Bitcoin network represents the first decentralized way to securely transfer value. But how can this added value of the network be assessed and related to its (social) cost to understand whether Bitcoin is an "efficient" or "inefficient" settlement layer?

While an absolute measure of this added value is hardly possible, we tackle this question in a relative way: We put the settled value on the Bitcoin network into perspective to 1 ) the costs of electricity consumption and 2) the resulting environmental damages caused by the electricity consumption of Bitcoin miners. This yields two running indices that quantify Bitcoin's value as a settlement layer with regard to 1) energy consumed and 2) social costs of carbon emission. These allow to compare the value of Bitcoin with other decentralized (blockchain) settlement layers, such as Ethereum [23], but also to contrast it with centralized settlement layers such as the American FedWire, Clearing House Interbank Payments System (CHIPS) or the European TARGET2 system. These systems which correspond most closely to a centralized equivalent of the Bitcoin network, since they represent base layers with final clearing. On this basis, a relative evaluation can be made as to what extent the Bitcoin network is more or less efficient or inefficient than its centralized counterparties.

This article proceeds as follows. In Section 2, the underlying data, assumptions and methods are listed. In Section 3, metrics and statistics on the Bitcoin network are presented, followed by the above-mentioned metrics for energy consumption and carbon emissions or social costs. Section 4 reflects on the main results and concludes.

\section{Data and variables}

The metrics developed in this article are based on different data, which in turn come from various sources. Table 1 provides an overview of the variables and respective data sources/references.

Table 1: Overview of variables.

\begin{tabular}{|c|c|c|}
\hline Variable & Description & Source \\
\hline \multicolumn{3}{|c|}{ Blockchain metrics } \\
\hline USD settled & $\begin{array}{l}\text { The amount of successfully transferred } \\
\text { Bitcoin transferred on the blockchain } \\
\text { multiplied with the price of Bitcoin in } \\
\text { USD. }\end{array}$ & [3] \\
\hline Transactions & $\begin{array}{l}\text { The amount of successful transactions } \\
\text { on the Bitcoin blockchain. }\end{array}$ & [3] \\
\hline \multicolumn{3}{|c|}{ Energy consumption and carbon footprint } \\
\hline BECl estimate & $\begin{array}{l}\text { The annualized estimation of Bitcoin's } \\
\text { energy consumption (in TWh). }\end{array}$ & [18] \\
\hline$B E C I$ minimum & $\begin{array}{l}\text { The annualized estimation of Bitcoin's } \\
\text { minimal energy consumption (in TWh). }\end{array}$ & [18] \\
\hline
\end{tabular}

\begin{tabular}{lll} 
CBECl estimate & $\begin{array}{l}\text { The annualized estimation of Bitcoin's } \\
\text { energy consumption (in TWh). }\end{array}$ & [19] \\
$\begin{array}{l}\text { CBECl lower } \\
\text { bound }\end{array}$ & $\begin{array}{l}\text { The annualized estimation of the lower } \\
\text { bound of Bitcoin's energy consumption } \\
\text { (in TWh). }\end{array}$ & {$[19]$} \\
$\begin{array}{l}\text { CBECl upper } \\
\text { bound }\end{array}$ & $\begin{array}{l}\text { The annualized estimation of the upper } \\
\text { bound of Bitcoin's energy consumption } \\
\text { (in TWh). }\end{array}$ & {$[19]$} \\
\hline
\end{tabular}

The first type of data (Bitcoin blockchain metrics) are accurately quantifiable metrics of the Bitcoin blockchain, which can be extracted from the blockchain in, e.g., daily, monthly or yearly intervals. The second category of data (Estimation of Bitcoin's energy consumption and carbon footprint) include estimates of energy consumption, energy mix, and carbon intensity of Bitcoin, which are accordingly subject to a certain degree of uncertainty. For this reason, we use two different data bases (the $\mathrm{CBECI}$ and $\mathrm{BECl}$ ), which are likely to be the most frequently cited sources in literature and media.

\section{Metrics for evaluating Bitcoin as a settle- ment layer.}

\subsection{Bitcoin network characteristics}

Figure 1 shows the four key metrics used in this research over time. It can be seen that both metrics used to estimate Bitcoin's energy consumption show a clear increasing trend. In 2017 Bitcoin miners consumed 6.6 TWh in energy and in 2020 already 66 TWh, and for 2021 the CBECl estimates it to be $130 \mathrm{TWh}$ [19]. $130 \mathrm{TWh}$ is equal to 130 billion kilowatt hours (kWh), a unit people are more familiar with. While the number of transactions remains comparatively stable over time, the USD settled per month shows a strong increase from the end of 2020. In early 2021, over 2.7 trillion USD are settled on the Bitcoin blockchain.

\subsection{Bitcoin's transactions settled in relation to its energy consumption}

We put the sum of Bitcoin transactions $\left(T X_{i, t}\right)$ in relation to the network's estimated energy consumption $\left(E C_{t}\right)$ :

$$
T X E_{t}=\frac{\sum T X_{i, t}}{E C_{t}}
$$

This allows us to quantify the basic efficiency of Bitcoin as a transaction network in relation to energy consumption. This relationship over time is visualized in Figure 2. For example, in January 2017 a transaction has cost $72 \mathrm{kWh}$, in January 2020 already $666 \mathrm{kWh}$ and in January 2021 it increased to $907 \mathrm{kWh}$ (all figures CBECl estimates). This increasing trend implies that the network has become less efficient in terms of energy efficiency for processing individual transactions. Or, as Bitcoin proponents would claim, that the network has become much more secure over time. 
(a) Estimated annualized energy consumption in TWh (BECl)

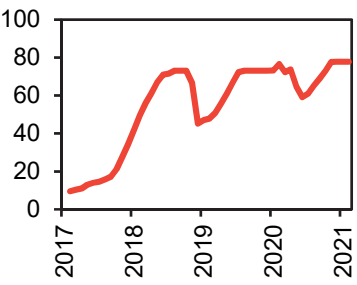

(c) USD billion settled per month

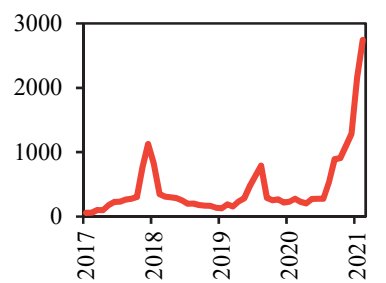

(b) Estimated annualized energy consumption in TWh (CBECl)

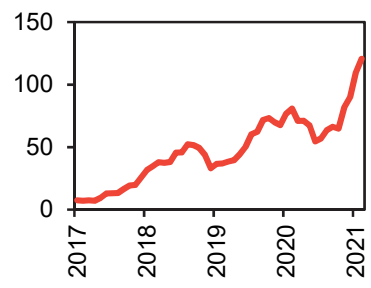

(d) Million transactions per month

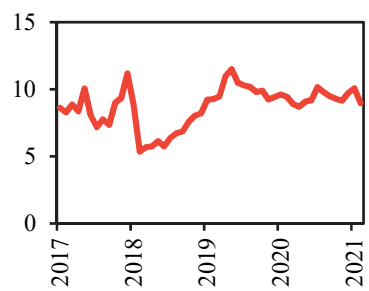

Fig. 1: Bitcoin network characteristics and estimates

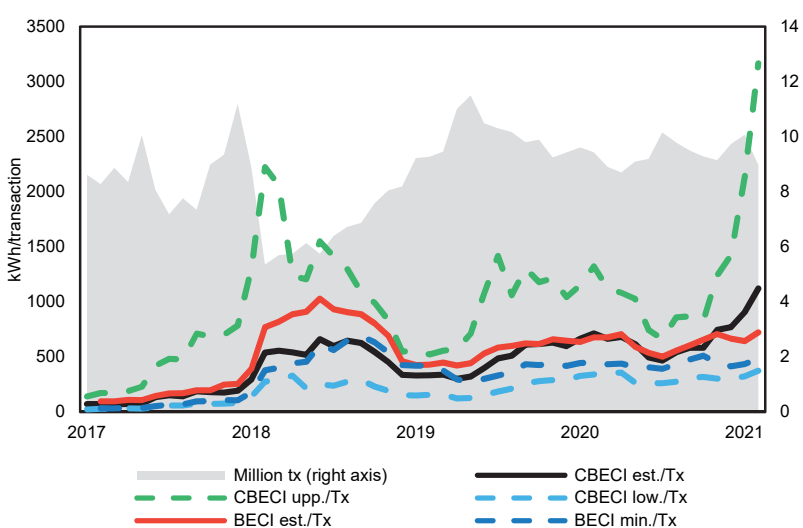

Fig. 2: Number of transactions settled in relation to different estimates of energy consumption of the Bitcoin network per month

However, missing from this consideration is the question of what exactly a transaction implies or means. For example, it makes a significant difference whether a transaction involves an equivalent value of $\$ 1$ or $\$ 1$ million. Accordingly, the equivalent value of transactions must be included in the calculation of the ratio to be meaningful.

\subsection{Bitcoin's value settled in relation to its energy consumption}

In the next step, we calculate the ratio between the transferred equivalent value of all Bitcoins and the estimated energy consumption of the network. We calculate the value as the product of the number of transferred Bitcoins $\left(B T C_{i, t}\right)$ with the traded Bitcoin price at the time of the transaction $\left(P(U S D)_{i, t}\right)$ :

$$
V S E_{t}=\frac{\sum\left(B T C_{i, t} * P(U S D)_{i, t}\right)}{E C_{t}}
$$

This relationship over time is visualized in Figure 3. Bitcoin settled $\$ 3,689$ billion in $2017, \$ 6,470$ billion in January 2020, and interpolated for 2021 will settle $\$ 29,138$ billion. Per kWh this translates to a settlement

of $\$ 211$ (BECl est.) to $\$ 278$ (CBECl est.) in 2017, around $\$ 92$ in 2020, and $\$ 252$ (CBECl est.) to $\$ 378$ (BECl est.) in 2021. Assuming a price of $\$ 0.05$ per $\mathrm{kWh}$ as a global average, this translates to a settlement of $\$ 4,220-\$ 5,560$ in $2017, \$ 1,840$ in 2020 and $\$ 5,040-\$ 7,560$ in 2021 per Dollar spent on energy. To put it differently, the transaction costs for a settled Dollar on the Bitcoin network decreased from $0.018 \%-0.024 \%$ in 2017 to $0.013 \%-0.020 \%$ in 2021.

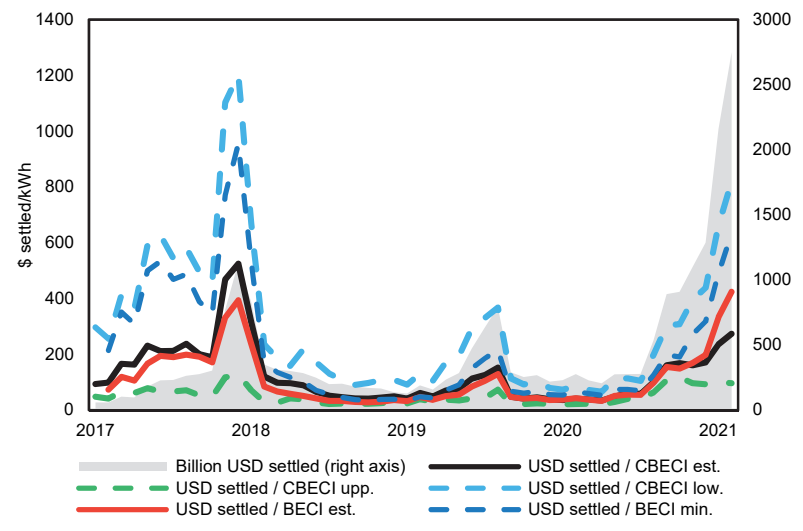

Fig. 3: USD value settled in relation to different estimates of energy consumption of the Bitcoin network per month

More detailed information on specific years as well as upper and lower bounds of energy consumption estimates are shown in Table 2.

\subsection{The social costs of Bitcoin's energy consump- tion}

The main argument against Bitcoin is not its energy consumption but rather the social costs caused by this consumption. Applying an estimated weighted average carbon intensity of 490 gCO2eq per kWh [12] allows to calculate the carbon footprint of the Bitcoin network. It yields $\mathrm{CO}_{2}$ emission of 6.51 ( $\mathrm{CBECl}$ est.) to 8.56 (BECl est.) million tons in 2017, around 34.5 million tons in 2020 and 38.13-57.17 million tons in 2021. With social costs of one emitted ton of carbon dioxide being estimated at $\$ 50$ [24], Bitcoin caused social costs of $\$ 0.33-0.43$ billion in 2017, $\$ 1.72$ billion in 2021, and will cost in 2021 between $\$ 1.91$ and $\$ 2.86$ in 2021.

These increasing social costs can now be put into perspective by comparing them with the Dollar settled (Figure 4). Back in 2017 the ratio of social costs to $\$$ settled is $0.009 \%$ to $0.012 \%$, in 2020 nearly $3 x$ higher with $0.027 \%$ and in 2021 lower again with $0.0065 \%-0.0097 \%$. 


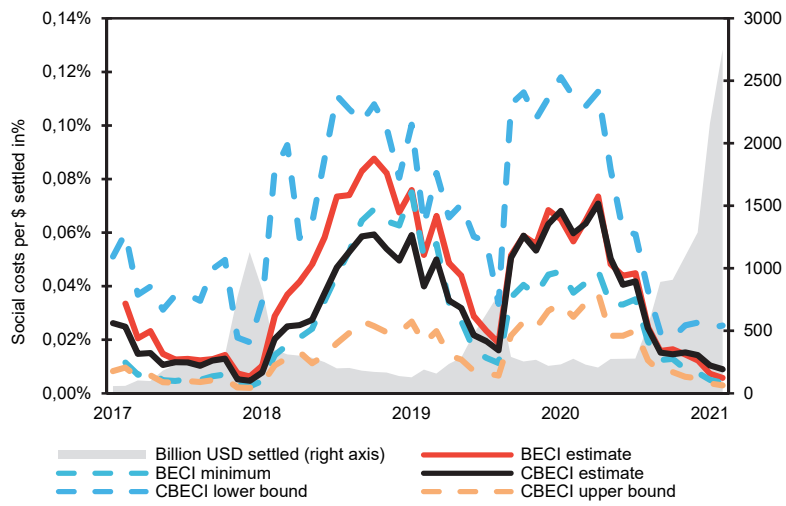

Fig. 4: Social costs per Dollar value settled for different estimates of energy consumption of the Bitcoin network per month.

\section{Concluding remarks}

The adoption and ownership of Bitcoin, cryptocurrencies and smart contract-based systems, such as decentralized finance (DeFi) or non-fungible tokens (NFTs) are becoming more and more relevant over time [25]-[28]. The increasing relevance of blockchain networks as a basic infrastructure for digital interaction and commerce poses a challenge for society to weigh the costs and benefits of this technology and innovation(s). There is no question that the Bitcoin network consumes a high amount of energy.

In this study, we contribute to the debate about the extent to which this energy consumption and its associated social costs of Bitcoin are "well spent" or "too high".

Table 2. Annual statistics on Dollar value settled in relation to energy consumption estimates of Bitcoin and the resulting social costs.

\begin{tabular}{|c|c|c|c|c|c|}
\hline & 2017 & 2018 & 2019 & 2020 & 2021 \\
\hline Value settled in billion USD & $3,689.38$ & $3,369.69$ & $3,922.07$ & $6,470.20$ & $29,398.48 i$ \\
\hline \multicolumn{6}{|l|}{ Bitcoin Energy Consumption Index (BECI) } \\
\hline Estimate in TWh & $17.47 i$ & 62.52 & 63.96 & 70.29 & 77.82 \\
\hline Minimum in TWh & $7.19 \mathrm{i}$ & 40.11 & 44.35 & 49.13 & 52.29 \\
\hline USD settled / estimate in kWh & 211.17 & 53.90 & 61.32 & 92.05 & 377.80 \\
\hline USD settled / minimum in kWh & 513.16 & 84.02 & 88.44 & 131.68 & 562.22 \\
\hline Carbon emission (estimate) in million tons $\mathrm{CO} 2$ & 8.56 & 30.63 & 31.34 & 34.44 & 38.13 \\
\hline Carbon emission (minimum) in million tons $\mathrm{CO} 2$ & 3.52 & 19.65 & 21.73 & 24.07 & 25.62 \\
\hline Costs of Bitcoin network (estimate) in billion USD & 0.43 & 1.53 & 1.57 & 1.72 & 1.91 \\
\hline Costs of Bitcoin network (minimum) in billion USD & 0.18 & 0.98 & 1.09 & 1.20 & 1.28 \\
\hline Social costs per Dollar settled (estimate) & $0.0116 \%$ & $0.0455 \%$ & $0.0400 \%$ & $0.0266 \%$ & $0.0065 \%$ \\
\hline Social costs per Dollar settled (minimum) & $0.0048 \%$ & $0.0292 \%$ & $0.0277 \%$ & $0.0186 \%$ & $0.0044 \%$ \\
\hline \multicolumn{6}{|l|}{ Cambridge Bitcoin Electricity Consumption Index (CBECI) } \\
\hline Estimate in TWh & 13.29 & 41.82 & 54.37 & 70.4 & 116.67 \\
\hline Lower bound in TWh & 5.35 & 18.93 & 23.58 & 34.33 & 39.71 \\
\hline Upper bound in TWh & 45.52 & 102.32 & 112.17 & 115.33 & 316.08 \\
\hline USD settled / estimate in kWh & 277.62 & 80.58 & 72.14 & 91.91 & 251.99 \\
\hline USD settled / lower bound in kWh & 689.10 & 178.05 & 166.35 & 188.46 & 740.39 \\
\hline USD settled / upper bound in kWh & 81.05 & 32.93 & 34.96 & 56.10 & 93.01 \\
\hline Carbon emission (estimate) in million tons $\mathrm{CO} 2$ & 6.51 & 20.49 & 26.64 & 34.50 & 57.17 \\
\hline Carbon emission (lower bound) in million tons $\mathrm{CO} 2$ & 2.62 & 9.28 & 11.55 & 16.82 & 19.46 \\
\hline Carbon emission (upper bound) in million tons $\mathrm{CO} 2$ & 22.30 & 50.14 & 54.96 & 56.51 & 154.88 \\
\hline Costs of Bitcoin network (estimate) in billion USD & 0.33 & 1.02 & 1.33 & 1.72 & 2.86 \\
\hline Costs of Bitcoin network (lower bound) in billion USD & 0.13 & 0.46 & 0.58 & 0.84 & 0.97 \\
\hline Costs of Bitcoin network (upper bound) in billion USD & 1.12 & 2.51 & 2.75 & 2.83 & 7.74 \\
\hline Social costs per Dollar settled (estimate) & $0.0088 \%$ & $0.0304 \%$ & $0.0340 \%$ & $0.0267 \%$ & $0.0097 \%$ \\
\hline Social costs per Dollar settled (lower bound) & $0.0036 \%$ & $0.0138 \%$ & $0.0147 \%$ & $0.0130 \%$ & $0.0033 \%$ \\
\hline Social costs per Dollar settled (upper bound) & $0.0302 \%$ & $0.0744 \%$ & $0.0701 \%$ & $0.0437 \%$ & $0.0263 \%$ \\
\hline
\end{tabular}

$\mathrm{i}$ : interpolated; Carbon emissions are in million tons based on 490 gCO2eq per kWh [12]; the cost per ton of CO2 is estimated at $\$ 50$ [24].

We argue that a differentiated approach is needed, which puts the resource consumption and the (social) costs of the settlement layer Bitcoin in a comparable context. For this purpose, we set different measures of Bitcoin's estimated energy consumption in relation to a) the settled value in Dollars and b) the social costs in the form of estimated carbon emissions. This allows us to determine key figures that enable comparability with classic settlement layers. The results show that in 2021, a single Dollar of (energy) cost enables a settled value between $\$ 5,040$ and $\$ 7,560$. This translates into average transaction costs of $0.013 \%-0.020 \%$.

This ratio increases with increasing power consumption and decreases with the Dollar amount settled. Since transactions are rather stable, the latter mainly relates to the price of Bitcoin, which is also the main driver of the hash power and thus energy invested into Bitcoin mining. It would thus not surprise, if both effects cancel each other and the social costs per transacted Dollar remains rather stable over time.

As a second metric to measure the relative efficiency of the Bitcoin network from a societal point of view, we suggest to compare the social costs resulting from carbon dioxide emissions of the Bitcoin mining activity to the value it settles every year. For every Dollar settled on the Bitcoin network in early 2021 , between $\$ 0.000065$ to $\$ 0.000097$ (or $0.065 \%$ to $0.0097 \%$ ) are caused in environmental damages due to $\mathrm{CO}_{2}$ emissions. This figure gives 
an indication that Bitcoin is indeed an expensive settlement layer, but that it is not totally out of line. It needs to be kept in mind that this result depends on the social cost of a carbon emission for which we used $\$ 50$ per ton. It also depends on the actual $\mathrm{CO}_{2}$ emission from Bitcoin mining. We simply assumed a global average, although there are good arguments that the ratio is higher for Bitcoin mining, which is often fueled by hydro power and an important contributor to shave peak loads and thus render renewable energies more efficient. However, it could also be argued that such energy is missing elsewhere and causes other energy demand to use non-renewables. Hence, the use of a global average seems adequate.

Potentially an even more informative evaluation of the efficiency of Bitcoin as a settlement layer comes from a comparison to other ways of settling value, for example, a bank wire or a payment in gold. This would need to take into account that the banking system hosts servers, maintains buildings, employs people (who not only need to commute to work, but also cannot engage in a different profession). The gold system comes with storage costs, costs of transportation and a mining process that is damaging to the environment and the workers, especially when quicksilver is used. However, to obtain the social costs of the banking system and the gold system are complex tasks beyond the scope of this paper.

Of course, it must be noted that there are significantly less energy-intensive blockchain networks or consensus mechanisms, which were not considered in more detail in this study [8]. Accordingly, future studies should not only include traditional settlement systems in their considerations and comparisons, but also other blockchains or distributed ledger systems.

\section{References}

[1] S. Nakamoto, "Bitcoin: A Peer-to Peer Electronic Cash System," 2008. [Online]. Available: https://bitcoin.org/bitcoin.pdf. [Accessed: 12-Jun-2019].

[2] Coingecko, “Bitcoin market capitalization," 2021. [Online]. Available: https://www.coingecko.com/en/coins/bitcoin. [Accessed: 10-Mar2021].

[3] Glassnode, "Glassnode.com," 2020. .

[4] BIS, "Foreign exchange turnover in April 2019 - Triennial Central Bank Survey," 2019.

[5] VISA, "VISA 2019 Corporate Responsibility \& Sustainability Report," 2020.

[6] The Federal Reserve, "Fedwire ${ }^{\circledR}$ Funds Service - Annual Statistics," 2021. [Online]. Available: https://www.frbservices.org/resources/financialservices/wires/volume-value-stats/annualstats.html. [Accessed: 15-Mar-2021].

[7] C. Dwork and M. Naor, "Pricing via processing or combatting junk mail," in Annual International Cryptology Conference, 1992, pp. 139-147.

[8] F. Steinmetz, L. Ante, and I. Fiedler, Blockchain and the Digital Economy: The Socio-Economic Impact of Blockchain Technology. Agenda Publishing, 2020.

[9] L. Ante, F. Steinmetz, and I. Fiedler, "Blockchain and energy: A bibliometric analysis and review," Renew. Sustain. Energy Rev., vol. 137, p. 110597, 2021.

[10] A. de Vries, "Bitcoin's Growing Energy Problem," Joule, vol. 2, no. 5, pp. 801-805, 2018.

[11] M. J. Krause and T. Tolaymat, "Quantification of energy and carbon costs for mining cryptocurrencies," Nat. Sustain., vol. 1, no. 11, pp. 711-718, 2018.

[12] C. Stoll, L. Klaaßen, and U. Gallersdörfer, "The Carbon Footprint of Bitcoin," Joule, vol. 3, no. 7, pp. 1647-1661, 2019.

[13] A. de Vries, "Bitcoin boom: what rising prices mean for the network's energy consumption," Joule, 2021.

[14] A. de Vries, "Bitcoin's energy consumption is underestimated: A market dynamics approach," Energy Res. Soc. Sci., vol. 70, p. 101721, 2020.

[15] J. Sedlmeir, H. U. Buhl, G. Fridgen, and R. Keller, "The Energy Consumption of Blockchain Technology: Beyond Myth," Bus. Inf. Syst. Eng., vol. 62, no. 6, pp. 599-608, 2020.

[16] U. Gallersdörfer, L. Klaaßen, and C. Stoll, “Energy Consumption of Cryptocurrencies Beyond Bitcoin," Joule, vol. 4, no. 9, pp. 1843-1846, 2020.

[17] J. Li, N. Li, J. Peng, H. Cui, and Z. Wu, "Energy consumption of cryptocurrency mining: A study of electricity consumption in mining cryptocurrencies," Energy, vol. 168, pp. 160-168, 2019.

[18] M. Rauchs, A. Blandin, and A. Dek, "Cambridge Bitcoin Electricity Consumption Index (CBECI)," 2021. .

[19] Digiconomist, "Bitcoin Energy Consumption Index," 2021. [Online]. Available: https://digiconomist.net/bitcoin-energy-consumption/. [Accessed: 05-Mar-2021].

[20] IEA, "Electricity Information: Overview," 2020. [Online]. Available: https://www.iea.org/reports/electricity-information-overview. [Accessed: 10-Mar-2021].

[21] E. Strehle and L. Ante, "Exclusive Mining of Blockchain Transactions," in Scientific Reports 2020 Conference proceedings of the Scientific Track of the Blockchain Autumn School 2020, 2020, pp. 8795.

[22] A. Back, "Hashcash - a denial of service countermeasure," 2002.

[23] V. Buterin, "Ethereum white paper - A next gerneration smart contract \& decentralized application platform," no. January, pp. 1-36, 2015.

[24] P. Howard and D. Sylvan, "Expert Consensus on the Economics of Climate Change," 2015.

[25] L. Ante, "Non-fungible token (NFT) markets on the Ethereum blockchain: Temporal development, cointegration and interrelations," BRL Work. Pap., vol. 22, 2021.

[26] F. Schär, "Decentralized Finance: On Blockchainand Smart Contract-based Financial Markets," Fed. 
Reserv. Bank St. Louis, 2021.

[27] L. Ante, "Smart Contracts on the Blockchain - A Bibliometric Analysis and Review," Telemat. Informatics, vol. 57, p. 101519, 2021.

[28] F. Steinmetz, M. von Meduna, L. Ante, and I. Fiedler, "Ownership, uses and perceptions of cryptocurrency: Results from a population survey," Technol. Forecast. Soc. Change, vol. 173, no. May, p. 121073, 2021. 